\title{
ORIGINAL
}

\section{ESTUDIO SOBRE TUBERCULOSIS EN UN DISTRITO SANITARIO DE SEVILLA. SITUACIÓN Y ALTERNATIVAS DE MEJORA EN EL CONTROL}

\section{Juan Limón Mora (1) y Pilar Nieto Cervera (2)}

(1) Servicios Centrales del Servicio Andaluz de Salud (SAS).

(2) Distrito Sanitario «Sevilla Este-Sur». Epidemiología y Programas.

\section{RESUMEN}

Fundamento: La situación actual en nuestro entorno (Distrito, Sevilla, Andalucía, España), donde no es raro observar incidencias anuales de TBC por encima de 30 casos nuevos por 100.000 habitantes, señala que el problema no está próximo, a ser erradicado. Este tantes, señala que el problema no está próximo, a ser erradicado. Este
trabajo tiene como objetivo describir los patrones clínicos y de salud pública de presentación de la tuberculosis en el ámbito del Distrito Sanitario «Sevilla Este-Sur».

Métodos: Estudio descriptivo de los casos de tuberculosis declarados (características personales, lugar, tiempo, tipo de enfermedad, factores de riesgos) durante el periodo 1992- 2000 en el distrito sanitario «Sevilla Este-Sur», situado en la ciudad de Sevilla y con algunos núcleos rurales. Se calculan la distribución porcentual de casos para los distintos factores estudiados y las tasas de incidencia en variables de interés (sexo, grupos edad, áreas geográficas).

Resultados: Se ha estimado una incidencia media anual de 19,4 casos / 100.000 habitantes. Existen diferencias en la incidencia po sexo $(R R=2,1)$, por grupos de edad (incidencia anual por encima de 24 casos 1100.000 habitantes en los niños de 0-4 y adultos 25-39 años de edad) y zona geográfica. Se observa la frecuencia de recidivas y repeticiones de tratamientos, así como carencias en la realización o comunicación de los estudios de familiares y contactos, observándose problemas de mal cumplimiento terapéutico y deficiencias de control en el entorno de los pacientes.

Conclusiones: El análisis de la situación en un distrito sanitario como el que se describe (alrededor de 610.000 habitantes en la actualidad) durante nueve años de seguimiento y con 1.065 casos declarados, puede orientar sobre una situación más general en nuestro entorno, posibilitando comparaciones con otros estudios. Se observa una tendencia descendente de la incidencia desde 1997. Se señalan algunas medidas organizativas a tener en cuenta para el control de la infección.

Palabras clave: Enfermedades infecciosas y parasitarias. Tuberculosis. Incidencia.

Correspondencia:

Juan Limón Mora

Servicios Centrales del SAS

Avda. de la Constitución 18

41071- Sevilla

Tfno: 955018510

Correo electrónico: jlimon@sc.sas.junta-andalucia.es

\section{ABSTRACT}

\section{Study on Tuberculosis in a Seville Healthcare District. Current Situation and Control Improvement Alternatives}

Background: In our setting (District, Seville, Andalusia, Spain, ...) it is not rare to find annual incidence rates for TBC of more than 30 new cases per 100,000 inhabitants. It reveals that this problem is nowhere near being eradicated. This study is aimed at describing the clinical and public health patterns of tuberculosis cases within the «South-East Seville» Healthcare District.

Methods: Descriptive study of the reported cases (personal traits, location, time, type of disease, risk factors) throughout the 1992-2000 period in the «South-East Seville» Healthcare District, located in the city of Seville and including some rural centers of population. Percentage distribution of cases for the different studied factors and the incidence rates for group of interest (sex, age groups, geographic areas) are provided.

Results: A mean annual incidence rate of 19.4 cases $/ 100,000$ inhabitants has been estimated. Notable differences in incidence depending on group of sex $(R R=2.1)$, age (annual incidence of more than 24 cases $/ 100,000$ inhabitants among children age $0-4$ and adults age 25-39) and geographical area were found. The frequency of relapses and repeated treatments, as well as shortcomings with regard to conducting and reporting the studies on family members and contacts were noted.

Conclusions: The situation analysis in a Healthcare District such as the one described here (currently approx. 610,000 inhabitants) throughout a nine-year follow-up period -entailing 1,065 reported cases- may well provide us some orientation about the situation in our environment. It also gives us the possibility to compare it with some other studies. A downward trend in the incidence rate has been noted as of 1997. Some organizational measures to be taken into account for controlling this infection are provided.
Key words: Communicable diseases. Tuberculosis. Inciden- 


\section{INTRODUCCIÓN}

Cuando parecía que la tuberculosis (TBC) es un problema de salud importante solo en los países en desarrollo, surgen unos condicionantes que hacen que, metidos en el siglo XXI, siga siendo objeto prioritario de atención para los sistemas sanitarios de los países industrializados. Se dispone de trabajos que presentan indicadores y valores de referencia de ámbito nacional ${ }^{1}$ que sirven para contrastar los datos obtenidos a un nivel local. Las personas infectadas por el VIH tienen un alto riesgo de padecer tuberculosis y la terapia debe ser ajustada a su estado inmunológico, siendo necesario establecer los mecanismos de vigilancia y análisis de información que pongan en relación el seguimiento de la tuberculosis y de la infección por el VIH, tanto a nivel nacional ${ }^{2}$ como en entornos más restringidos ${ }^{3}$. La multiresistencia a los fármacos antituberculosos es un fenómeno en ascenso que debe ser monitorizado y sobre el que sólo se dispone de trabajos puntuales ${ }^{4}$. Por otra parte, no se confía en disponer con prontitud de nuevas drogas eficaces y los tratamientos estándar se vuelven en muchos casos inefectivos. El cumplimiento del tratamiento por parte de los afectados y el seguimiento clínico de la terapia deja mucho que desear. Son necesarias medidas especiales de control y surgen trabajos epidemiológicos sobre la tuberculosis en distintos contextos, como escuelas ${ }^{5,6}$, prisiones ${ }^{7}$ y colectivos especialmente afectados, como las personas adictas a drogas 3 o los inmigrantes ${ }^{8}$. Todas estas cuestiones relacionadas hacen que cobre importancia disponer de un sistema integrador de vigilancia y control que no se limite a recoger información sino que, trabajando en la misma organización, asegure continuidad en el proceso de recogida de información e intervención individualizada, familiar y colectiva.

Los esquemas tradicionales de trabajo a demanda dentro de los centros sanitarios y sin definición clara de responsabilidades y tareas, hacen que estemos chocando contra un problema en el que es necesario el trabajo comunitario, muchas veces sin la colaboración del paciente. Igualmente es necesaria una organización que asegure integralidad y seguimiento (en la actualidad, la organización sanitaria tiene bastantes limitaciones en la conexión entre niveles asistenciales, instituciones y estamentos profesionales). Habría que concretar más la coordinación y definir responsabilidades claras, dotando de recursos a equipos que aborden el problema de la forma más integral y autosuficiente posible.

Este trabajo tiene como objetivo describir los patrones clínicos y de salud pública de presentación de la tuberculosis en el ámbito del Distrito Sanitario «Sevilla Este-Sur», donde confluyen la atención primaria y especializada para el diagnóstico, tratamiento y seguimiento de los pacientes. Este trabajo también quiere profundizar en el análisis de la situación organizativa asistencial y de Salud Pública, intentando brindar alternativas con vistas a la mejora del control de esta enfermedad

\section{MATERIAL Y MÉTODOS}

La base fundamental de las observaciones que se presentan en este trabajo parten del registro de Enfermedades de Declaración Obligatoria (EDO), en el que se han venido recogiendo de forma continuada, y relativamente estable, los casos nuevos (incidencia) de tuberculosis. Habitualmente se realiza una declaración en el momento del diagnóstico, siendo poco frecuente la declaración posterior realizada por el mismo médico sobre datos de evolución. Asimismo, va siendo cada vez más inusual la declaración anticipada o de sospecha de la simple infección tuberculosa, esperándose habitualmente a completar el estudio (cultivos, radiología,...) para realizar la notificación una vez confirmado el caso. Se han clasificado los casos notificados, recogidos en ficheros 
informatizados, y se han evitado duplicidades y otros problemas de registro.

Se presentan los datos descriptivos en un formato sencillo de frecuencias, porcentajes que supone cada categoría de las distintas variables y, en su caso, cálculo de la tasa de incidencia de la enfermedad en relación a la población afectada (por grupos de edad, sexo, zona de salud y sección censal). Al tratarse de un registro que pretende ser exhaustivo recogiendo todas las enfermedades tuberculosas que se produzcan, no hemos realizado estimaciones de significación estadística de las diferencias encontradas, utilizable cuando se trabaja sobre muestras representativas de una población general a estudio. Como denominadores poblacionales se han utilizado el censo y el padrón de habitantes realizado en 1996 (a mitad del periodo en estudio). Además del Distrito en su conjunto, se han estudiado otras unidades territoriales pequeñas, presentándose el análisis de la incidencia acumulada en el periodo (1992-2000), y calculando tasas medias anuales dividiendo la tasa global del periodo por los nueve años de seguimiento. Los cálculos de incidencia año a año solamente se presentan para el total del Distrito.

Este trabajo ha presentado la dificultad y el interés de seguir la aparición de casos en un territorio amplio, compuesto a su vez de una serie de unidades territoriales más pequeñas. Se ha estudiado la incidencia acumulada en nueve años de seguimiento de 29 Zonas Básicas de Salud (con una media de unos 21.000 habitantes). Hasta el año 1998, cuando se unificó todo el territorio como distrito sanitario único, existían dos distritos diferenciados: «Sur» 16Zonas Básicas ( 5 de las cuales comprendían municipios fuera de la ciudad de Sevilla), y «Este» con 13 Zonas Básicas de Salud, todas ellas urbanas o integradas en la ciudad.

En la parte Este de la ciudad de Sevilla, ha sido factible realizar el análisis más detallado por sección censal (unidad territorial de unos 3.500 habitantes por término medio). El mapa que se dibuja tras el análisis y representación espacial de la información (programa informático EPIMAP) permite detectar de forma sencilla los lugares de mayor prioridad de intervención para el control, y facilita la visualización de agrupaciones de territorios cercanos o colindantes. Asimismo, se ha prestado atención al estudio de las agrupaciones de casos familiares, muchas veces dilatadas en el tiempo, apareciendo casos secundarios en una misma familia años después de la aparición del primer caso. Todas estas agrupaciones son importantes a la hora de priorizar actividades de trabajo en los centros sanitarios y demandar que se realicen labores de control en un ámbito comunitario.

\section{RESULTADOS}

En el periodo estudiado fueron notificados un total de 1.065 casos de TBC en personas residentes en el territorio del distrito, lo que supone una incidencia media anual de 19,4/100.000 habitantes. En las tablas 1 a 4 se presentan con detalle los resultados descriptivos para el periodo 1992-2000 sobre todas las cuestiones referentes a distribución de casos (en el tiempo, territorio, característica personales, características de la enfermedad, riesgos y datos sobre estudio de convivientes).

En tabla 1, se puede observar la tendencia anual en la notificación de casos de tuberculosis, apreciándose el máximo en el año 1996 con un descenso mantenido a partir de 1997. Mes a mes se observa que la declaración de casos es bastante más baja en los meses de agosto, noviembre y diciembre, observándose un patrón estacional con meses de mayor y menor actividad diagnóstica (durante el primer semestre se diagnosticaron, sumando todo el periodo, el $57 \%$ de los casos frente al $43 \%$ de la segunda mitad del año).

En la tabla 2, se aprecia la notificación de aproximadamente dos tercios de los casos en 
Tabla 1

Frecuencia variables descriptivas. Distribución en el tiempo y según características personales

\begin{tabular}{|c|c|c|}
\hline & número de casos & $\%$ \\
\hline \multicolumn{3}{|l|}{$A \tilde{n} o$} \\
\hline 1992 & 88 & 8,3 \\
\hline 1993 & 74 & 6,9 \\
\hline 1994 & 102 & 9,6 \\
\hline 1995 & 145 & 13,6 \\
\hline 1996 & 168 & 15,8 \\
\hline 1997 & 150 & 14,1 \\
\hline 1998 & 129 & 12,1 \\
\hline 1999 & 90 & 8,5 \\
\hline 2000 & 119 & 11,2 \\
\hline Total & 1065 & 100,0 \\
\hline \multicolumn{3}{|l|}{ Mes } \\
\hline 1 Enero & 77 & 8,7 \\
\hline 2 & 78 & 8,8 \\
\hline 3 & 94 & 10,6 \\
\hline 4 & 77 & 8,7 \\
\hline 5 & 88 & 10,0 \\
\hline 6 & 89 & 10,1 \\
\hline 7 & 67 & 7,6 \\
\hline 8 & 52 & 5,9 \\
\hline 9 & 84 & 9,5 \\
\hline 10 & 75 & 8,5 \\
\hline 11 & 51 & 5,8 \\
\hline 12 Diciembre & 52 & 5,9 \\
\hline Total & 884 & 100,0 \\
\hline no consta & 181 & \\
\hline Total & 1.065 & \\
\hline
\end{tabular}

varones (riesgo relativo de 2,1). Al analizar por grupos de edad se observan las tasas de incidencia más elevadas entre los 25 y 39 años, centrándose el máximo a los 32 y 33 años.

En la tabla 3 se presenta la distribución de casos por zona básica de salud, apreciándose diferencias territoriales según residencia de los afectados. A partir de algunos indicadores básicos se han comparado las características de las pequeñas unidades territoriales (secciones censales) donde se han observado tasas de incidencia más elevadas (por encima de 30/100.000 habitantes y año), respecto a valores globales o de referencia en su
Tabla 2

Incidencia media anual por sexo y grupo de edad

\begin{tabular}{|lccc|}
\hline & $\begin{array}{c}\text { número } \\
\text { de casos }\end{array}$ & $\%$ & $\begin{array}{c}\text { incidenciamedia } \\
\text { anual } / 10^{5} \text { hab. }\end{array}$ \\
\hline Sexo & & & \\
\hline hombre & 721 & 68,0 & 26,9 \\
mujer & 339 & 32,0 & 12,1 \\
Total & 1065 & 100,0 & 19,4 \\
\hline grupos de edad & & \\
\hline & & & \\
$0-4$ años & 53 & 5,2 & 24,5 \\
$5-9$ & 50 & 4,9 & 17,4 \\
$10-14$ & 12 & 1,2 & 3,6 \\
$15-19$ & 45 & 4,4 & 10,2 \\
$20-24$ & 82 & 8,0 & 15,5 \\
$25-29$ & 150 & 14,7 & 30,7 \\
$30-34$ & 170 & 16,7 & 36,8 \\
$35-39$ & 108 & 10,6 & 27,1 \\
$40-49$ & 138 & 13,5 & 20,2 \\
$50-59$ & 85 & 8,3 & 14,3 \\
$60-69$ & 66 & 6,5 & 12,6 \\
$70-90$ & 60 & 5,9 & 11,2 \\
Total & 1065 & 100,0 & 19,4 \\
\hline
\end{tabular}

entorno. Así se puede observar el porcentaje de personas «analfabetas o sin estudios» y el porcentaje de personas que alcanzan a realizar «estudios superiores» entre las personas que residen en las secciones que presentan mayor incidencia.

Entre los 400 casos notificados en la parte del distrito denominada «Sevilla Este», se detecta y declara acumulación de dos o más casos de enfermedad tuberculosa en 42 familias o grupos de convivientes. En el estudio de los contactos, habitualmente familiares, se detectaron 29 familias con dos casos, 9 familias con tres casos y cuatro familias con cuatro casos cada una (nos referimos siempre a casos de enfermedad, sin incluir los casos de simple infección tuberculosa que haya podido haber). Es decir, en más del $10 \%$ de los enfermos se ha producido una acumulación familiar de casos simultánea o diferida en el tiempo.

En la tabla 4 se pueden revisar los datos referentes a la distribución según características de la enfermedad. La mayor parte 
Tabla 3

Distribución geográfica por zonas básicas de salud y secciones

\begin{tabular}{|c|c|c|}
\hline Zona Básica de Salud & número de casos & incidencia media anual $/ 10^{5}$ habitantes \\
\hline no consta & 33 & \\
\hline Alcosa & 24 & 11,3 \\
\hline Candelaria & 58 & 31,2 \\
\hline Centro & 97 & 48,6 \\
\hline Cerro del Águila & 52 & 26,4 \\
\hline Coria del rio & 88 & 43,6 \\
\hline El porvenir & 49 & 23,5 \\
\hline E1 Tardón & 10 & 8,1 \\
\hline Greco & 36 & 23,3 \\
\hline Huerta del rey & 8 & 4,3 \\
\hline Isla mayor & 1 & 1,6 \\
\hline Juan XXIII & 22 & 18,6 \\
\hline Juncal & 18 & 8,4 \\
\hline La plata & 32 & 20,6 \\
\hline Las Letanias & 44 & 32,8 \\
\hline Los remedios & 14 & 5,6 \\
\hline Mairena del Aljarafe & 37 & 12,9 \\
\hline Montequinto & 20 & 13,0 \\
\hline Nervión & 9 & 6,7 \\
\hline Polígono Aeropuerto & 17 & 5,3 \\
\hline Poligono sur & 159 & 86,3 \\
\hline Puebla del rio & 7 & 9,0 \\
\hline San Jacinto & 1 & 0,5 \\
\hline San Juan de Aznalfarache & 73 & 30,6 \\
\hline San Pablo & 30 & 13,8 \\
\hline Santa Genoveva & 3 & 2,5 \\
\hline Santa Teresa & 18 & 8,8 \\
\hline Sector sur & 1 & 0,8 \\
\hline Torreblanca & 47 & 24,5 \\
\hline Triana & 53 & 28,5 \\
\hline
\end{tabular}

SECCIONES CENSALES con mayor incidencia o aparición de nuevos casos «Sevilla Este»). Características demográficas (*) y tasa de incidencia media anual de «enfermedad tuberculosa».

\begin{tabular}{|c|c|c|c|c|c|}
\hline «código» & $\begin{array}{c}0-14 \text { años } \\
(\%)\end{array}$ & $\begin{array}{c}\text { Mayor } 65 \text { años } \\
(\%)\end{array}$ & $\begin{array}{l}\text { Analfabeto o sin } \\
\text { estudios }(\%)\end{array}$ & $\begin{array}{c}\text { Estudios superiores } \\
(\%)\end{array}$ & $\begin{array}{c}\text { Tasa media anual } / 10^{5} \\
\text { habitantes }\end{array}$ \\
\hline «406» & 19,8 & 16,9 & 39,3 & 0,5 & 43,2 \\
\hline «429» & 21,4 & 13,5 & 34,7 & 0,1 & 68,1 \\
\hline «456» & 20,4 & 7,5 & 34,7 & 0,9 & 42,5 \\
\hline «458» & 27,6 & 6,0 & 36,7 & 0,2 & 30,2 \\
\hline «462» & 35,1 & 8,1 & 64,0 & 0 & 39,6 \\
\hline «421» & 15,7 & 16,8 & 36,3 & 1,2 & 60,2 \\
\hline$« 467 »$ & 28,8 & 5,6 & 20,8 & 1,6 & 30,9 \\
\hline «361» & 11,8 & 17,8 & 29,2 & 1,0 & 99,9 \\
\hline «471» & 28,6 & 5,0 & 38,1 & 0,3 & 30,2 \\
\hline Valor referencia & 20,0 & 11,6 & 23,6 & 4,0 & 19,4 \\
\hline
\end{tabular}

(*) Porcentajes de personas según grupos de edad y nivel de estudios en las distintas Secciones.

Datos del Padrón Municipal año 1996. Valor de referencia es el valor medio del conjunto de las secciones censales de Sevilla «Este». 
Tabla 4

Frecuencia variables descriptivas. Características de la enfermedad

\begin{tabular}{|c|c|c|}
\hline & número de casos & $\%$ \\
\hline \multicolumn{3}{|l|}{ situación evolutiva } \\
\hline Inicial & 419 & 86,0 \\
\hline Recidiva & 52 & 10,7 \\
\hline Retratamiento & 16 & 3,3 \\
\hline Total & 487 & 100,0 \\
\hline no consta & 578 & \\
\hline Total & 1065 & \\
\hline \multicolumn{3}{|l|}{ confirmación del caso } \\
\hline no confirmado & 218 & 25,2 \\
\hline cultivo o baciloscopia + & 647 & 74,8 \\
\hline Total & 865 & 100,0 \\
\hline no consta & 200 & \\
\hline Total & 1065 & \\
\hline \multicolumn{3}{|l|}{ baciloscopia } \\
\hline Positivo & 400 & 70,8 \\
\hline Negativo & 144 & 25,5 \\
\hline no hecho & 21 & 3,7 \\
\hline Total & 565 & 100,0 \\
\hline no consta & 500 & \\
\hline Total & 1065 & \\
\hline \multicolumn{3}{|l|}{ cultivo } \\
\hline Positivo & 264 & 80,2 \\
\hline Negativo & 33 & 10,0 \\
\hline no hecho & 32 & 9,7 \\
\hline Total & 329 & 100,0 \\
\hline no consta & 736 & \\
\hline Total & 1065 & \\
\hline \multicolumn{3}{|l|}{ localización } \\
\hline Pulmonar & 769 & 84,2 \\
\hline Pleural & 34 & 3,7 \\
\hline Meníngea & 8 &, 9 \\
\hline Otras & 102 & 11,2 \\
\hline Total & 913 & 100,0 \\
\hline no consta & 152 & \\
\hline Total & 1065 & \\
\hline \multicolumn{3}{|l|}{ tratamiento } \\
\hline estándar (2ZRH/4RH) & 417 & 73,0 \\
\hline no estándar & 154 & 27,0 \\
\hline Total & 571 & 100,0 \\
\hline no consta & 494 & \\
\hline Total & 1065 & \\
\hline
\end{tabular}

de las tuberculosis declaradas en las que aparece esta información son enfermedades en estadio «inicial» $(86 \%)$. Se señala hasta un $14 \%$ de recidivas y retratamientos entre los casos donde esta información se anota. 
La confirmación de los casos mediante baciloscopia y/o cultivo se realizó en tres cuartas partes de los enfermos, habiendo entre los restantes estudios de confirmación no bacteriológica, frecuente sobretodo en niños (radiología, anatomía patológica...). Entre las baciloscopias realizadas (544) se obtiene un $70,8 \%$ de resultados positivos, valor que se acerca a la sensibilidad de la prueba (dado que todos los pacientes son considerados con enfermedad tuberculosa, aunque puede haber un porcentaje de ellos no baciliferos). Si seleccionamos los casos de «tuberculosis pulmonar» (769) se eleva la sensibilidad de la prueba en este grupo hasta el $81,7 \%$, y si además lo restringimos a los mayores de 14 años (por las dificultades para la obtención correcta de esputo en niños) sube hasta el 82,4\%. Los resultados del cultivo se anotan solo en una reducida proporción de casos $(27,9 \%)$, dado el largo tiempo que suele transcurrir desde el diagnóstico inicial y la obtención de este resultado. Para el total de casos (297 cultivos) se obtiene una sensibilidad del $88,9 \%$, elevándose a 93,2 \% si el paciente tiene enfermedad «pulmonar» y 94,6 \% si además es mayor de 14 años.

La localización de la enfermedad es mayoritariamente pulmonar $(84,2 \%)$. Las formas graves de tuberculosis meníngea $(0,9$ $\%$ ) y diseminada, aunque raras, están presentes y hay que tenerlas en cuenta para valorar la importancia de este problema de salud. Asimismo el tratamiento más frecuente que se instaura es el estándar (73\%) recomendado en el Consenso Nacional sobre Tuberculosis $^{9}$ ( 2 meses de isoniazida, rifampicina y pirazinamida, continuando con 4 meses de los dos primeros fármacos). $\mathrm{Al} 27$ $\%$ restante de los pacientes se les prescribe un tratamiento distinto, en muchos casos debido a problemas de recidiva, resistencias o patologías añadidas.

En tabla 5 se describe la frecuencia de otros procesos asociados a la enfermedad tuberculosa y situaciones de especial vigilancia. La seropositividad VIH/SIDA (15,5
$\%)$, adicción a drogas parenterales $(9,9 \%)$ y alcoholismo $(5,1 \%)$ son los factores de riesgo más frecuentes entre los notificados. También se recoge el número de enfermos convivientes, señalándose hasta un 14,1\% de pacientes en los que hay agrupación conocida de casos de enfermedad tuberculosa en su entorno. La frecuencia de simple infección tuberculosa asintomática en el entorno del paciente se recoge en cerca del $40 \%$ de los estudios realizados y notificados.

\section{DISCUSIÓN}

La incidencia media anual fue de 19,4 casos/100.000 habitantes calculada para el periodo 1992-2000, observándose un patrón descendente a partir de 1997, quizás coincidente con el observado para la mortalidad por sida. Complementario de los datos presentados en resultados, para el periodo 19941996 se obtuvo información sobre casos detectados (a veces no notificados) en distintos niveles y laboratorios del área. Tanto en atención primaria como especializada, se hizo un rescate activo de casos en los distintos laboratorios y se indagó en los archivos de historias clínicas. Así se pudo hacer, por primera vez, una estimación más afinada y completa, que la que suministraba solamente la notificación de casos a través de las Enfermedades de Declaración Obligatoria. Se estimó una incidencia anual en distrito «Sevilla Este» de 30,9 casos/100.000 habitantes, durante 1994. Por el mismo procedimiento se estimó la incidencia anual en el distrito «Sevilla Sur» siendo las tasas de 35,25 y 36,85 durante los años 1995 y 1996 respectivamente. Estos niveles, podríamos decir endémicos de aparición de nuevos casos, siguen siendo elevados al compararlos con los países de nuestro entorno.

La tasa media anual de declaración de casos, en concreto la del año 2000 en el distrito (19,4 por 100.000 habitantes), está muy cercana a la recogida a nivel nacional $(19,6$ 
Tabla 5

Frecuencia variables descriptivas. características referentes a factores de riesgo de los casos y estudio de convivientes

\begin{tabular}{|c|c|c|}
\hline & número de casos & $\%$ \\
\hline \multicolumn{3}{|l|}{ factores de riesgo } \\
\hline VIH/SIDA & 165 & 15,5 \\
\hline ADVP & 105 & 9,9 \\
\hline Alcoholismo & 54 & 5,1 \\
\hline ninguno conocido & 103 & 9,7 \\
\hline Vagabundo & 6 & ,6 \\
\hline interno prisión & 8 & ,8 \\
\hline institución cerrada & 9 & ,9 \\
\hline Inmigrante & 3 &, 3 \\
\hline profesión de riesgo & 3 &, 3 \\
\hline Desnutrición & 21 & 2,0 \\
\hline otra patología o riesgo & 77 & 7,2 \\
\hline Total pacientes & 1065 & \\
\hline \multicolumn{3}{|c|}{ número de convivientes estudiados en cada uno de los casos } \\
\hline 1 & 47 & 11,4 \\
\hline 2 & 59 & 14,3 \\
\hline 3 & 98 & 23,8 \\
\hline 4 & 73 & 17,7 \\
\hline 5 & 31 & 7,5 \\
\hline 6 & 35 & 8,5 \\
\hline $7-8$ & 33 & 8,0 \\
\hline $9-12$ & 18 & 4,3 \\
\hline$>12$ & 17 & 4,1 \\
\hline Total & 412 & 100,0 \\
\hline no consta o ningún conviviente & 653 & \\
\hline Total & 1065 & \\
\hline \multicolumn{3}{|l|}{ número de enfermos convivientes } \\
\hline 0 & 457 & 85,9 \\
\hline 1 & 41 & 7,7 \\
\hline 2 & 29 & 5,5 \\
\hline 3 & 4 &, 8 \\
\hline 4 & 1 &, 2 \\
\hline Total & 532 & 100,0 \\
\hline no consta o ningún conviviente & 533 & \\
\hline Total & 1065 & \\
\hline \multicolumn{3}{|l|}{ número de infectados convivientes } \\
\hline 0 & 311 & 60,2 \\
\hline 1 & 70 & 13,5 \\
\hline 2 & 66 & 12,8 \\
\hline 3 & 35 & 6,8 \\
\hline 4 & 14 & 2,7 \\
\hline 5 & 9 & 1,7 \\
\hline$>5$ & 12 & 2,4 \\
\hline Total & 517 & 100,0 \\
\hline no consta o ninguno conocido & 548 & \\
\hline Total & 1065 & \\
\hline
\end{tabular}


casos TBC por 100.000)1. Igualmente la distribución de los casos según sexo es prácticamente idéntica a la observada para el conjunto de España (el número de casos en hombres suponen el doble del de las mujeres en ambos casos), que también se observa en otras publicaciones con fuente de información y metodología similar ${ }^{10}$.

El descenso de la incidencia de tuberculosis a partir de 1997 se asemeja también al observado a nivel nacional, pudiendo ir en paralelo con la tendencia descendente observada desde mitad de los noventa para la infección VIH/Sida ${ }^{2}$.

En cuanto a la incidencia según la edad hay que señalar que los grupos con tasas más elevadas corresponden a adultos jóvenes y niños (25-40 años, 0-9 años). Por otra parte, se observa en preescolares una incidencia doble que en escolares; a partir de los siete años la incidencia desciende notablemente, siendo hasta esa edad más probable encontrar casos en seguimiento y detección precoz de salud infantil. Además, se observa una baja detección en el distrito sanitario de nuevos casos de TBC en personas mayores, al compararlo con las cifras nacionales ${ }^{1}$. Mientras que a nivel del Estado se observan tres veces más casos en personas mayores de 65 años que en niños de 0-14 años, en Sevilla las cifras en ambos grupos son similares. Las razones pueden ser múltiples (carencias en la detección de casos en personas mayores; excesos de diagnósticos sin confirmación microbiológica en niños; diferencias reales en ambos grupos...). Habría que estudiar en profundidad las causas de estas diferencias resolviendo eventuales problemas de búsqueda activa y diagnóstico de casos.

Según datos del Estudio Multicéntrico para el estudio de la tuberculosis en 13 Comunidades Autónomas en España ${ }^{5}$ el riesgo anual de infección (RAI) medido durante el curso escolar 1996-1997 fue aproximadamente de $0,1 \%$ para la cohorte de niños de 6 años y $0,3 \%$ para los de 14 años.
Por tanto, en este y otros estudios españoles ${ }^{6}$ se observa una tendencia favorable al descenso del riesgo de infección en niños en los últimos años. En el distrito sanitario puede ser conveniente contrastar esta información, disponiéndose en alguna zona de salud de datos históricos, en medio escolar, para poder hacerlo.

En tabla 3 se puede ver el estudio demográfico de los barrios o secciones censales del Distrito Sanitario «Sevilla Este». Así se puede observar el porcentaje de personas «analfabetas o sin estudios» y el porcentaje de personas que alcanzan a realizar «estudios superiores» entre las personas que residen en cada un de las 9 secciones con mayor incidencia. Según el Padrón de habitantes en esos barrios o secciones territoriales, vemos cifras que hablan comparativamente del muy bajo nivel de estudios de sus habitantes (cifras medias de $37,1 \%$ de personas catalogadas como «analfabeto o sin estudios»y solo $0,7 \%$ de personas con «estudios superiores», para valores de referencia en el conjunto de la ciudad de $23,6 \%$ y $4 \%$ respectivamente).

Recientemente se ha puesto en evidencia, a través de estudios geográficos o ecológicos, la relación entre pobreza y resurgimiento de la tuberculosis en barrios de ciudades en países desarrollados, persistiendo la asociación incluso después de ajustar factores como la distinta incidencia de SIDA, proporción de inmigración y raza ${ }^{11}$. Parece necesario implantar programas priorizados de actuación y recursos en ciertas zonas y servicios sanitarios.

La mayor parte de las tuberculosis declaradas en las que aparecen estos datos, son enfermedades en estadio «inicial» $(86 \%)$. En cuanto a la localización, hasta el 84,2\% son pulmonares, coincidiendo este porcentaje con algún gran estudio que muestra cifras muy similares ${ }^{12}$.

Al $27 \%$ de los enfermos se les prescribe un tratamiento no estándar, en muchos casos 
debido a problemas de recidiva o retratamientos (14\% en nuestro caso), patologías añadidas como el VIH/sida (hasta 15,5\% recogido en este trabajo), o resistencias a antimicrobianos, todos ellos factores importantes para el control individual y comunitario del problema. En estudio realizado en pacientes con cultivo positivo a Mycobacterium tuberculosis en Zaragoza se señala hasta un $10,9 \%$ de resistencias al menos a un fármaco antituberculoso, y un 2,3\% de multiresistencias al menos a rifampicina e isoniazida ${ }^{4}$.

A través de estudios descriptivos basados en notificación de casos es bastante complicado hacer estimaciones fiables sobre la frecuencia de ciertas características de los enfermos que no siempre son conocidas o comunicadas (consumo de drogas, estado serológico en relación al VIH, internamientos...). Por otra parte las comparaciones en las cifras con otros estudios son bastante arriesgadas, al ser difícil normalizar los criterios de recogida y medida. En nuestro país se dispone de estudios parciales sobre distintos colectivos de enfermos (prisiones ${ }^{7}$, inmigrantes $^{8}, \mathrm{ADVP}^{3}$ ). Esta subnotificación puede afectar con más intensidad a grupos de gran interés en salud pública (interno en prisión, en instituciones cerradas, vagabundo, inmigrante, profesión de riesgo).

En este estudio se constatan limitaciones en la realización y estudio completo de los convivientes y contactos de los enfermos. El número de personas estudiadas en los estudios de contactos es relativamente bajo, quedando con frecuencia convivientes sin estudiar. Se han analizado las agrupaciones familiares de casos, a veces muy dilatadas en el tiempo, y que el Sistema de Vigilancia Epidemiológica detecta siendo habitual que no sean notificadas o recordadas. Estas agrupaciones son importantes a la hora de priorizar actividades de trabajo en los centros sanitarios y demandar que se realicen labores de control en un ámbito comunitario.
Para el control de la tuberculosis en nuestro entorno, en pocas ocasiones se han llegado a concretar verdaderos programas, con actividades definidas y una dotación de recursos que garantice los compromiso adoptados. Actualmente hay medidas eficaces y efectivas de control de TBC, pero hay que desarrollarlas en cada entorno. Algunas de estas medidas implican modificaciones organizativas de los centros y tareas de los profesionales, como son:

- Dotar a los médicos de los centros de salud de formación en salud pública, para el desarrollo de programas preventivos. Definición clara de tareas para control de TBC y otras cuestiones de salud, para llevarlas a cabo de forma sistemática y reglada día a día.

- Igualmente, habría que ampliar el espectro de problemas de salud atendidos en las actuales consultas de enfermería y atención domiciliaria (en TBC supervisión de los tratamientos, captación, estudio del foco de la infección y seguimiento de las quimioprofiláxis; labor de consejo y asistencia VIH/ sida...).

- Los fármacos antituberculosos, pensamos que deberían ser gratuitos y suministrados al enfermo como tratamiento supervisado por el sanitario que le atiende directamente. Esto es especialmente importante en poblaciones con bajos recursos, que son las más susceptibles de padecer la tuberculosis.

- Conseguir que cada uno de los servicios especializados desarrolle el trabajo de área que le corresponde potenciando la coordinación y el reparto de responsabilidades con atención primaria.

- Responsabilidades, en algunas cuestiones ineludibles e intrasladables, para los estamentos superiores de la organización sanitaria: 
- Planificación de red de laboratorios que asegure mínimos de cobertura y estabilidad (incluyendo laboratorios de salud pública). Estos laboratorios deben estar conectados y coordinados, y deben cubrir de forma equitativa el conjunto necesidades de asistencia individualizada y de salud pública.

- Desarrollo normativo que apoye y clarifique las actuaciones profesionales $\mathrm{y}$ deberes de salud pública.

- Compromisos efectivos entre organismos e instituciones (Instituciones Penitenciarias, Ayuntamientos, Educación, Asuntos sociales,..) para el seguimiento de pacientes durante todo el proceso de la enfermedad.

\section{AGRADECIMIENTOS}

A todas las personas que trabajan en el Sistema de Vigilancia Epidemiológica en el distrito y asistencia especializada. Agradecimiento especial para María Luisa Sierra y Manuel Cardero, que siempre nos ayudaron técnicamente y llevan gran parte del trabajo en la gestión diaria de los sistemas de información.

\section{BIBLIOGRAFÍA}

1. Rodríguez Valín E. Situación actual de la tuberculosis en España: Incidencia y mortalidad desde 1995. Características de los casos de tuberculosis y meningitis tuberculosa declarados en 2000. Bol Epidemiol Semanal 2001; 9: 293-304.

2. Vigilancia epidemiológica del Sida en España. Situación a 30 de Junio de 2001. Bol Epidemiol Semanal 2001; 9: 181-185.

3. Alvarez Rodríguez M, Godoy Garcia P. Prevalencia de tuberculosis e infección VIH entre participantes en un programa de control para usuarios de drogas intravenosas. Rev Esp Salud Pública. 1999;73:375-81.

4. Torres L, Arazo P, Blas Perez J, del Pilar Amador M, Antonia Lezcano M, Jose Revillo M, Bautista García-Moya J. Resistencia del Mycobacterium tuberculosis en Zaragoza, España (1993-1997) y factores relacionados. Med Clin (Barc) 2000;115:605-9.

5. Alcaide Megías J, Altet Gómez MN, Canela i Soler J. Epidemiología de la tuberculosis. An Esp Pediatr. 2000;53:449-57.

6. Urbina Torija J, Garcia Salazar M, Ruiz Perez R, Cecilia Villamor A, Martínez Perez J, Sinde Masa J. Prevalencia de la infección tuberculosa a edad escolar en Guadalajara, España. Gac Sanit 2000;14:110-6.

7. Martín V, Brugos M, Valcárcel I. Prevalencia de la infección tuberculosis en una prisión provincial. Rev Esp Salud Publica 2000;74:361-6.

8. Rivas-Clemente FP, Nacher-Conches M, Corrillero-Martín J, Vélez-Reyes S, Huerta-Galindo L. Resultados de un programa de screening activo para tuberculosis en inmigrantes procedentes del Maghreb: aceptabilidad y adherencia. Aten Primaria 1999;24:411-6.

9. FIS. Ministerio de Sanidad y Consumo. Consenso Nacional para el control de la tuberculosis en España. Med Clin (Barc) 1992; 98: 24-31.

10. Martín v, Alonso MA, Ramos J, Otero A, Cortizo J, Travieso S. Incidencia de la tuberculosis respiratoria en la provincia de León según el sistema de notificación de enfermedades de declaración obligatoria,1992-1999. Rev Esp Salud Pública 2002; 76 : 239-48.

11. Barr RG, Diez-Roux AV, Knirsch CA, Pablos-Méndez A. Neighborhood poverty and the resurgence of tuberculosis in New York City, 1984-1992. Am J Public Health 2001;91:1487-93.

12. De Riemer K, Moreira FM, Werneck Barreto AM, Ueleres Braga J. Survey of mycobacteriology laboratory practices in an urban area with hyperendemic pulmonary tuberculosis. Int J Tuberc Lung Dis 2000;4:776-83. 